\title{
Anatomic distribution of supraclavicular lymph node in patients with esophageal cancer
}

This article was published in the following Dove Press journal:

OncoTargets and Therapy

22 September 2016

Number of times this article has been viewed

\section{Jun Xing' \\ Yijun Luo ${ }^{1,2}$ \\ Xiaoli Wang ${ }^{1,2}$ \\ Min Gao' \\ Mingping Sun \\ Xiuping Ding' \\ Tingyong Fan' \\ Jinming $\mathrm{Yu}^{\prime}$}

'Department of Radiation Oncology and Radiology, Shandong Cancer Hospital Affiliated to Shandong University, ${ }^{2} \mathrm{School}$ of Medical and Life Sciences, Shandong Academy of Medical Sciences, University of Jinan, Jinan, People's Republic of China
Correspondence: Jinming Yu Department of Radiation Oncology and Radiology, Shandong Cancer Hospital Affiliated to Shandong University, Jiyan Road 440, Jinan 250I 17, Shandong,

People's Republic of China

Email sdyujinming@|26.com
Purpose: Definitive chemoradiation therapy remains the standard of care for patients with localized esophageal carcinoma who choose nonsurgical management. However, there is no consensus regarding delineation of the nodal clinical target volume (CTVn), especially for lower cervical lymph nodes. This study aimed to map the location of metastatic supraclavicular lymph nodes in thoracic esophageal carcinoma patients with supraclavicular node involvement and generate an atlas to delineate the CTVn for elective nodal radiation of esophageal squamous cell carcinoma.

Patients and methods: In this study, the supraclavicular regional lymph node was further divided into four subgroups. The locations of the involved supraclavicular nodes for all patients were then transferred onto a template computed tomography (CT) image. A volume probability map was then generated with nodal volumes, and was displayed on the template CT to provide a visual impression of nodal frequencies and anatomic distribution.

Results: We identified 154 supraclavicular nodal metastases based on CT image in 96 patients. Of these, $29.2 \%$ were located in group I region, $59.7 \%$ in group II region, $10.4 \%$ in group III region, and $0.7 \%$ in group IV region.

Conclusion: On the basis of our study, we suggest that the appropriate radiation field of CTVn should include the group I and II regions and the CTVn exterior margin along the lateral side of the internal jugular vein may be suitable.

Keywords: esophageal carcinoma, lymph node metastasis, clinical target volume, cervical lymph node

\section{Introduction}

Esophageal cancer (EC) is the sixth leading cause of cancer-related death worldwide and is characterized by extreme aggression and poor prognosis. ${ }^{1,2}$ Cervical and upper thoracic lesions account for $\sim 15 \%$ of all ECs. A complicated anatomy limits the surgical procedures that can be used for these lesions. ${ }^{3}$ Definitive chemoradiation therapy (DCRT) remains the standard of care for patients with localized EC who choose nonsurgical management. ${ }^{4}$ Radiation therapy, a component of DCRT, is one of the most important therapeutic methods in the treatment of the upper thoracic esophageal squamous cell carcinoma (ESCC). Most studies report that the neck and upper mediastinum are the regions most commonly affected by nodal metastasis in patients with upper thoracic EC. ${ }^{5-7}$ Ding et al surveyed 18,415 patients in 45 observational studies to determine which node level should be included in the target volume for patients undergoing DCRT. ${ }^{8}$ Their data came from patients who were undergoing surgical treatment with two-field or three-field dissection. The lymph node metastasis rates of upper thoracic EC in the cervical, upper mediastinal, mid-mediastinal, lower mediastinal, and abdominal levels were $30.7 \%, 42.0 \%, 12.9 \%, 2.6 \%$, and $9 \%$, 
respectively. Similarly, Li et al demonstrated that for cervical and upper thoracic lesions, the most common site of nodal metastasis is the neck (39.2\% and $21.8 \%$, respectively), and proposed that the supraclavicular lymph nodes should be included at the superior border of the target volume. ${ }^{9}$ Guidelines from the US National Comprehensive Cancer Network recommend that for cervical EC, the supraclavicular nodes and even the higher echelon cervical nodes should be subjected to elective treatment. ${ }^{10}$

However, there is no consensus regarding delineation of the nodal clinical target volume (CTVn), especially for lower cervical lymph nodes. Esophageal carcinoma is greatly capable of metastasizing with an extensive and not clearly defined range of invasion. ${ }^{11}$ Taking into consideration the microscopic spread, the irradiation fields of nodal target volume may cover both involved lymph nodes and regional nodes. Of note, a large lateral extent of the conventional supraclavicular irradiation field theoretically provides a better regional control, while the radiotherapy-related toxicities cannot be ignored and the implementation of three-dimensional conformal radiotherapy and intensitymodulated radiation therapy requires accurate and precise definition of the CTVn. ${ }^{12-15}$

In this study, we characterize the distribution of these nodes according to computed tomography (CT) data in patients with supraclavicular regional lymph node involvement, and use this evidence to suggest contours of the appropriate clinical target volume (CTV) margin in supraclavicular nodal region, which would potentially improve conformal treatment in this patient population with decreased treatmentrelated toxicity.

\section{Materials and methods Patients}

After obtaining approval from the Medical Ethics Committee of Shandong Cancer Hospital Affiliated to Shandong University, we retrospectively reviewed our database of upper ESCC patients from June 2010 to July 2015. All patients had been histologically or cytologically confirmed with ESCC. We retrospectively analyzed patients who had cervical and upper thoracic ESCC without distant metastasis at diagnosis. Esophageal lesions in the reviewed patients were assessed and characterized using examinations that included esophagography, endoscopy, CT imaging, and for some patients, combined positron-emission tomographycomputed tomography and endoscopic ultrasonography. The eligible criteria for inclusion in the study were a diagnosis of metastatic supraclavicular regional lymph nodes, either relapse at the supraclavicular region after chemoradiation with involved field or initial metastases, and availability of diagnostic CT scans of the supraclavicular region. Clinical characteristics of 96 EC patients with supraclavicular node involvement are shown in Table 1. All participants provided written informed consent.

\section{CT diagnosis of metastatic nodes}

All CT images were reviewed and interpreted by two experienced radiological experts. Positive lymph nodes in our study were included based on the fluorodeoxyglucose avidity and CT appearance of the node. Features supporting the consideration for metastasis included a short axis $>10 \mathrm{~mm}$, a round shape, inhomogeneous enhancement, the presence of necrosis, at locations uncommon for reactive nodes, fluorodeoxyglucose avidity (if tested), and obviously responsive to anticancer treatment. ${ }^{16-18}$ Only those with qualified CT images explicitly exhibiting the sizes and locations of malignant nodes in the whole range of the supraclavicular region and lower neck were included.

\section{Definition of each lymph node group and node mapping}

The supraclavicular region is divided into the left and right sides of the body midline, and in this study, each side was further divided into four subgroups based on the imaging-based classification for the cervical nodes reported by Som et al or Feng et al including para-recurrent laryngeal neural nodes (group I), para-internal jugular venous

Table I Clinical characteristics of 96 esophageal cancer patients with supraclavicular node involvement

\begin{tabular}{ll}
\hline Characteristics & Value \\
\hline Age & 62 \\
Median & $42-81$ \\
Range & \\
Sex & 72 \\
Male & 24 \\
Female & \\
Primary tumor & 33 \\
Cervical & 63 \\
Upper thoracic & \\
Stage & 10 \\
I & 16 \\
II & 57 \\
III & 13 \\
IV & \\
Diagnosis of SCV node involvement & 10 \\
Initial presentation & 86 \\
Relapse at follow-up & \\
Treatment intention & 91 \\
Curative & 5 \\
Palliative & \\
\hline
\end{tabular}

Abbreviation: SCV, supraclavicular. 
Table 2 The anatomic features of each lymph node station in the supraclavicular zone

\begin{tabular}{|c|c|}
\hline Level & Boundary \\
\hline Para-recurrent laryngeal & Upper border: lower margin of the cricoid cartilage \\
\hline \multirow[t]{6}{*}{ neural lymph nodes (I L, I R) } & Lower border: upper border of the manubrium \\
\hline & Anterior border: anterior border of the carotid artery; posterior border of the trachea \\
\hline & Posterior border: anterior border of the vertebral body \\
\hline & Lateral border: medial border of the carotid artery; lateral border of the vertebral body \\
\hline & Medial border: the midline \\
\hline & The midline of the trachea serves as the border between I R and I L \\
\hline Para-internal jugular venous & Upper border: lower margin of the cricoid cartilage \\
\hline \multirow[t]{6}{*}{ lymph nodes (II L, II R) } & Lower border: upper border of the manubrium \\
\hline & Anterior border: anterior border of internal jugular vein \\
\hline & Posterior border: anterior border of transverse process \\
\hline & Lateral border: lateral border of the internal jugular vein; anterior and medial borders \\
\hline & of the anterior scalene muscle \\
\hline & Medial border: medial border of the carotid artery; lateral border of the vertebral body \\
\hline Supraclavicular lymph nodes & Upper border: upper border of the subclavian artery \\
\hline \multirow[t]{6}{*}{ (III L, III R) } & Lower border: upper border of the manubrium \\
\hline & Anterior border: posterior border of sternocleidomastoid \\
\hline & Posterior border: front edge of the anterior scalene muscle \\
\hline & Lateral border: posterior border of sternocleidomastoid; posterior and lateral borders \\
\hline & of the anterior scalene muscle \\
\hline & Medial border: lateral border of the carotid artery \\
\hline Posterior cervical lymph & Upper border: lower margin of the cricoid cartilage \\
\hline nodes (IV L, IV R) & Lower border: upper border of the manubrium; posterior edge of the \\
\hline & sternocleidomastoid \\
\hline
\end{tabular}

Abbreviations: L, left; $R$, right.

nodes (group II), supraclavicular lymph nodes (group III), and level $\mathrm{Vb}$ posterior cervical lymph nodes (group IV). Table 2 shows the details of the anatomic structures of interest and the reference blood vessels for each lymph node region. ${ }^{19,20}$

The locations of the involved supraclavicular nodes for all patients were then transferred onto a template $\mathrm{CT}$ image (registered by hand drawings from the diagnostic CT scan onto the template $\mathrm{CT}$ scan) according to methods previously described. ${ }^{21,22}$ The volumetric center of each lymph node was identified and used for subsequent characterization of location. All mapped lymph node locations were individually reviewed and registered most precisely onto the template $\mathrm{CT}$ image, based on the location in the original CT image, by a radiologist and by two radiation oncologists. A volume probability map was then generated with nodal volumes, and was displayed on the template $\mathrm{CT}$ for a visual representation of the axial density of distribution. Finally, CTV contours of the suggested supraclavicular regional nodes were generated by radiation oncologists specialized in treatment of esophageal carcinoma, based on the anatomical distribution.

\section{Results}

\section{Patient characteristics}

A total of 96 cervical EC and upper thoracic esophageal carcinoma patients with supraclavicular lymph node metastasis were retrospectively examined at our institution from June 2010 to July 2015. The sample included 72 male patients and 24 female patients with a median age of 62 years (range, $42-81$ years). Of these, ten patients had stage I, 16 had stage II, 57 had stage III, and 13 had stage IV disease. In the 96 patients, 154 nodes (the number of positive lymph nodes in each patient ranged from 1-5) were considered to be metastatic in the supraclavicular region, 79 on the left and 75 on the right.

\section{Anatomic distribution of supraclavicular nodes}

The distribution of 154 supraclavicular nodal metastases in 96 patients is shown in Figure 1. We further evaluated in detail the lymph node involvement frequency for each lymph node group. In patients with upper EC, the lymph node spread affected mainly the group I and group II regions. Of the 154 nodes, $29.2 \%$ were located in group I region, $59.7 \%$ in group II region, $10.4 \%$ in group III region, and $0.7 \%$ in group IV region. The metastatic characteristics of lymph nodes in the subgroup supraclavicular region are shown in Table 3.

\section{Suggested CTVn according to the results}

We generated an atlas that serves as a template for contouring the supraclavicular nodal volumes at risk in patients with upper esophageal carcinoma who adopted elective nodal irradiation (ENI). 

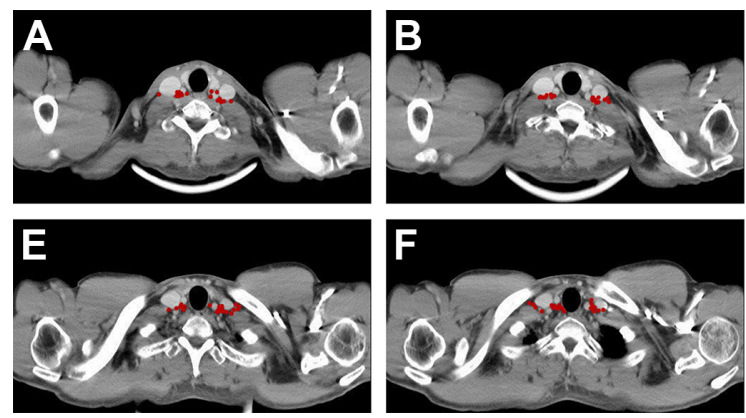
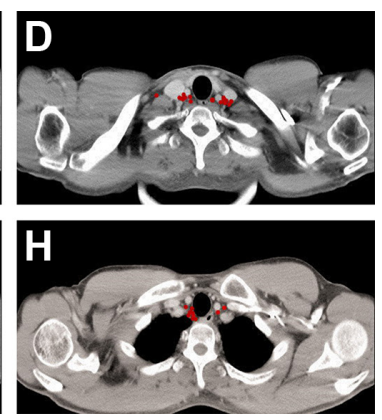

Figure I The volumetric center of each lymph node was identified and used for subsequent characterization of location on the template.

Notes: Figures $(\mathbf{A}-\mathbf{H})$ represent CT images from the annular cartilage to the entrance of the thorax in order to show the supraclavicular region, with an axial sampling thickness of $5 \mathrm{~mm}$ per slice. Representative axial images (superior to inferior) depicting the location of I54 supraclavicular lymph nodes.

Abbreviation: CT, computed tomography.

On the basis of our study, the lower cervical lymph nodes of the group I and group II regions are considered to be the high-risk regions of lymph node metastasis, which were defined as suitable for ENI. Of note, $10.4 \%$ of involved lymph nodes were located in group III region, and those positive lymph nodes were close to the lateral side of the internal jugular vein. Approximately $99 \%$ of involved lymph nodes in lower cervical region could be safely encompassed by a CTVn, including lymph node metastasis in group I, group II, and group III regions, indicating that the CTVn exterior margin along the lateral side of the internal jugular vein may be suitable.

\section{Discussion}

Chemoradiotherapy has an important role in the treatment of EC in both the inoperable and the preoperative settings. However, regarding the target volume for radiation, different clinical practices exist. To date, the use of ENI or involved-field radiotherapy for chemoradiotherapy is still controversial. Some investigators consider that an irradiation volume covering only the gross tumor is appropriate. When using ENI or involved-field radiotherapy, primary tumor recurrence and distant metastases are the common failure sites, and the overall survival does not differ. ${ }^{23}$ However, some study revealed that the use of ENI can significantly increase local control and reduce regional nodal relapse, and therefore prolong the overall survival.

Table 3 Distribution of lower cervical nodal metastases

\begin{tabular}{llll}
\hline Subgroup & Right side & Left side & Total \\
\hline I & 23 & 22 & 45 \\
II & 43 & 49 & 92 \\
III & 8 & 8 & 16 \\
IV & I & 0 & I \\
Total & 75 & 79 & I54 \\
\hline
\end{tabular}

If prophylactic irradiation therapy is considered, accurate delineation of CTV is critical in the effective management of ESCC. Accurate delineation may improve the probability of local control and reduce the risk of complications. Currently, information on the location of supraclavicular regional lymph nodes is lacking. The optimal radiotherapy target volume for the supraclavicular lymph node region has not been determined. Because of inadequate data on the anatomical distribution of involved lymph nodes, patients treated with a supraclavicular field usually have no evidence of gross supraclavicular disease, and target volumes are often defined primarily on the basis of the distribution of normal lymphatics or by vascular and bony landmarks that have traditionally been used for field design.

To address the limitation of the current studies, we describe the distribution of supraclavicular region lymph nodes in patients with thoracic esophageal carcinoma according to CT imaging in our institution and analyzed the anatomic distribution of supraclavicular region lymph nodes in patients to outline the spatial and probabilistic distributions of supraclavicular region lymph nodes, providing data for an evidence-based approach to supraclavicular region radiation therapy field design. As seen in our study, the atlas showed that metastasis frequently occurred in the lower cervical lymph nodes in group I, group II, and group III regions. Combining the anatomy with our data, those regions are considered to be at high risk of ESCC lymph node metastasis, which were defined as areas suitable for ENI. Approximately $89 \%$ of involved lymph nodes in supraclavicular region could be safely encompassed by a CTVn, including group I and group II regions. The incidence of supraclavicular lymph node involvement in the group III region was $10.4 \%$, and the location of those lymph nodes was close to the lateral side of the internal jugular vein. Moreover, the incidence of lymph node involvement in 
group IV was low, and it may be reasonable to exclude these lymph nodes from the CTVn. On the basis of our study, for patients with upper thoracic ESCC who choose DCRT as their treatment, we suggest that the appropriate radiation field of CTVn should include the group I and II regions and the CTVn exterior margin along the lateral side of the internal jugular vein may be suitable.

However, there are several limitations in our current study. First, our findings are based on an interpretation of CT imaging and not on pathology assessments. Microscopic disease can occur in normal-sized lymph nodes, and lymph node enlargement can be caused by benign conditions, limiting the accuracy of CT for interpreting nodal involvement in EC to $39 \%-85 \%{ }^{24}$ In addition, according to a meta-analysis, CT showed $50 \%$ sensitivity and $83 \%$ specificity for determining regional lymph node metastasis in thoracic tumors. ${ }^{25}$ Secondly, disadvantages in node registration by hand drawing onto one template CT are inherent. Additionally, there is a small, inherent source of error in transferring the involved supraclavicular nodes from each individual patient to a template CT. Finally, ours was a retrospective study with a small sample size, and included only 96 patients with supraclavicular regional lymph node involvement, which yielded 154 lymph nodes for the present analysis. Fortunately, the location of these nodes was highly reproducible, as illustrated by the volume probability maps in Figure 1, suggesting that our sample size is sufficient to propose the CTV contours of supraclavicular nodes.

Despite our study's limitations, we believe that our study contributes novel, patient population-based data regarding the anatomic distribution of positive supraclavicular regional lymph nodes to generate a contour that may serve as a "template" to guide CTV delineation during conformal treatment planning. Our proposed contours may result in improved targeting of radiation treatment, which may allow for a significant decrease in normal tissue irradiation and its associated toxicities.

\section{Conclusion}

In summary, safe use of precise radiotherapy to prevent regional lymph node metastasis depends on accurate identification of the CTVn. We used CT imaging to create a probability map that sets out the incidence of nodal involvement by anatomic group in patients with EC. This study provides the radiation oncologist with valuable information regarding the anatomic distribution of supraclavicular metastases, which may serve as a "template" to guide CTV delineation for ENI of upper thoracic ESCC and might result in improvements to the target contour during radiation treatment, with decreased treatment-related toxicity.

\section{Disclosure}

The authors report no conflicts of interest in this work.

\section{References}

1. Torre LA, Bray F, Siegel RL, Ferlay J, Lortet-Tieulent J, Jemal A. Global cancer statistics, 2012. CA Cancer J Clin. 2015;65(2):87-108.

2. Ferlay J, Shin HR, Bray F, Forman D, Mathers C, Parkin DM. Estimates of worldwide burden of cancer in 2008: GLOBOCAN 2008. Int $J$ Cancer. 2010;127(12):2893-2917.

3. Koshy M, Esiashvilli N, Landry JC, Thomas CR Jr, Matthews RH. Multiple management modalities in esophageal cancer: epidemiology, presentation and progression, work-up, and surgical approaches. Oncologist. 2004;9(2):137-146.

4. Stahl M, Budach W, Meyer HJ, Cervantes A. Esophageal cancer: Clinical Practice Guidelines for diagnosis, treatment and follow-up. Ann Oncol. 2010;21 Suppl 5:v46-v49.

5. Chen J, Liu S, Pan J, et al. The pattern and prevalence of lymphatic spread in thoracic oesophageal squamous cell carcinoma. Eur $J$ Cardiothorac Surg. 2009;36(3):480-486.

6. Jang HJ, Lee HS, Kim MS, Lee JM, Zo JI. Patterns of lymph node metastasis and survival for upper esophageal squamous cell carcinoma. Ann Thorac Surg. 2011;92(3):1091-1097.

7. Huang W, Li B, Gong H, et al. Pattern of lymph node metastases and its implication in radiotherapeutic clinical target volume in patients with thoracic esophageal squamous cell carcinoma: a report of 1077 cases. Radiother Oncol. 2010;95(2):229-233.

8. Ding X, Zhang J, Li B, et al. A meta-analysis of lymph node metastasis rate for patients with thoracic oesophageal cancer and its implication in delineation of clinical target volume for radiation therapy. Br J Radiol. 2012;85(1019):e1110-e1119.

9. Li M, Liu Y, Xu L, et al. Computed tomography-based distribution of involved lymph nodes in patients with upper esophageal cancer. Curr Oncol. 2015;22(3):e178-e182.

10. [No authors listed] Prescribing, recording, and reporting photon-beam intensity-modulated radiation therapy (IMRT): contents. $J$ ICRU. 2010;10(1):NP.

11. Komaki R, Liao Z, Forster K, Lee HK, Stevens CW, Cox JD. Target definition and contouring in carcinoma of the lung and esophagus. Rays. 2003;28(3):225-236.

12. Cooper JS, Guo MD, Herskovic A, et al. Chemoradiotherapy of locally advanced esophageal cancer: long-term follow-up of a prospective randomized trial (RTOG 85-01). Radiation Therapy Oncology Group. JAMA. 1999;281(17):1623-1627.

13. Minsky BD, Pajak TF, Ginsberg RJ, et al; INT 0123 (Radiation Therapy Oncology Group 94-05) phase III trial of combined-modality therapy for esophageal cancer: high-dose versus standard-dose radiation therapy. J Clin Oncol. 2002;20(5):1167-1174.

14. Zhao KL, Shi XH, Jiang GL, Wang Y. Late-course accelerated hyperfractionated radiotherapy for localized esophageal carcinoma. Int $J$ Radiat Oncol Biol Phys. 2004;60(1):123-129.

15. Wang S, Liao Z, Chen Y, et al. Esophageal cancer located at the neck and upper thorax treated with concurrent chemoradiation: a single-institution experience. $J$ Thorac Oncol. 2006;1(3): 252-259.

16. Xu Y, Liu J, Du X, et al. Prognostic impact of postoperative radiation in patients undergoing radical esophagectomy for pathologic lymph node positive esophageal cancer. Radiat Oncol. 2013;8:116.

17. Hsu PK, Huang CS, Wang BY, Wu YC, Hsu WH. Survival benefits of postoperative chemoradiation for lymph node-positive esophageal squamous cell carcinoma. Ann Thorac Surg. 2014;97(5): 1734-1741. 
18. Liu Q, Cai XW, Wu B, Zhu ZF, Chen HQ, Fu XL. Patterns of failure after radical surgery among patients with thoracic esophageal squamous cell carcinoma: implications for the clinical target volume design of postoperative radiotherapy. PLoS One. 2014;9(5):e97225.

19. Som PM, Curtin HD, Mancuso AA. An imaging-based classification for the cervical nodes designed as an adjunct to recent clinically based nodal classifications. Arch Otolaryngol Head Neck Surg. 1999; 125(4):388-396.

20. Feng ZX, Zhao LJ, Guan Y, et al. Identification of risk factors and characteristics of supraclavicular lymph node metastasis in patients with small cell lung cancer. Med Oncol. 2013;30(1):493.

21. Brown LC, Diehn FE, Boughey JC, et al. Delineation of supraclavicular target volumes in breast cancer radiation therapy. Int $J$ Radiat Oncol Biol Phys. 2015;92(3):642-649.
22. Jing H, Wang SL, Li J, et al. Mapping patterns of ipsilateral supraclavicular nodal metastases in breast cancer: rethinking the clinical target volume for high-risk patients. Int J Radiat Oncol Biol Phys. 2015; 93(2):268-276.

23. Li M, Zhang X, Zhao F, Luo Y, Kong L, Yu J. Involved-field radiotherapy for esophageal squamous cell carcinoma: theory and practice. Radiat Oncol. 2016;11:18.

24. van Vliet EP, Heijenbrok-Kal MH, Hunink MG, Kuipers EJ, Siersema PD. Staging investigations for oesophageal cancer: a metaanalysis. Br J Cancer. 2008;98(3):547-557.

25. de Langen AJ, Raijmakers P, Riphagen I, Paul MA, Hoekstra OS. The size of mediastinal lymph nodes and its relation with metastatic involvement: a meta-analysis. Eur J Cardiothorac Surg. 2006;29(1): 26-29.
OncoTargets and Therapy

\section{Publish your work in this journal}

OncoTargets and Therapy is an international, peer-reviewed, open access journal focusing on the pathological basis of all cancers, potential targets for therapy and treatment protocols employed to improve the management of cancer patients. The journal also focuses on the impact of management programs and new therapeutic agents and protocols on

\section{Dovepress}

patient perspectives such as quality of life, adherence and satisfaction The manuscript management system is completely online and includes a very quick and fair peer-review system, which is all easy to use. Visit http://www.dovepress.com/testimonials.php to read real quotes from published authors. 